\title{
Routing Optimization in WBAN using Bees Algorithm for Overcrowded Hajj Environment
}

\author{
Ghassan Ahmed Ali*, Shah Murtaza Rashid Al Masud \\ College of Computer Sciences and Information systems, \\ Najran University, Najran, Kingdom of Saudi Arabia
}

\begin{abstract}
Crowded places like Hajj environment in Makkah which host from 2 to 3 million on specific area and time can pose health challenges for pilgrims who need medical care. One of the solutions to overcome such difficulties is to use Wireless Body Area Networks (WBANs). WBAN is one of the new technology using wireless sensor network to gather data about status of patient then to forward collected data to be proceeded. However, various types of challenges in WBAN should be concerned. Power consumption is critical within WBAN system. Furthermore, delay of data transfer may lead to wrong diagnosis or uncorrected report that may lead to death; therefore, the transferred data must be reliable to ensure accuracy in measurement. In this paper, we propose a framework for routing optimization in medical wireless network. The proposed framework optimize shortest path in different stages of collected data to get less energy consumption, and reduce transmission time. The proposed work is based on Bees Algorithm to overcome such challenges and find shortest path for data within shortest time during overcrowded of Hajj environment. Matlab simulation results show good performance of Bees Algorithm in terms of reducing transmission time, energy consumption, delay, and throughput.
\end{abstract}

Keywords-Wireless Body Area Network (WBAN); Bees algorithm; routing optimization; Hajj environment

\section{INTRODUCTION}

Bees Algorithm is inspired by the foraging behavior of honeybee in nature to find the best solution of a given optimization problem proposed by [1]. According to [2], Bees Algorithm approved its higher performance compared to many heuristic optimization in many problems. As a result of its simplicity, Bees Algorithm applied in many application including solving examination timetabling problems [3], training neural networks [4], job scheduling for machine [5], supply chain optimization [6], data clustering [7], correlationaware service in cloud [8], and robot path in dynamic environment [9]. It is obvious that the study of Bees Algorithm and its applications in the literature increases exponentially. The Bees Algorithm shows its efficiency in terms of speed, learning, and accuracy.

In this paper, Bees Algorithm is used as optimization method to assist in deployment of WBAN and make transmission of WBAN more efficient during Hajj. The Hajj is an annual pilgrimage to Makkah in Saudi Arabia. It is performing once-in-a-lifetime obligation for all Muslims who have the ability to undertake the journey. Two to three millions pilgrims from different countries are gathering and the number of pilgrims is increasing every year. One of the most challenges is that the complete events of Hajj must be performed in a specific locations not exceed $4 \mathrm{~km}^{2}$ within specific 5 days. Therefore, places become over-crowded and difficult to be reached by medical emergency in case of injuries. However, there are some public's health care centers surrounding Makkah, but because most of pilgrims are not familiar with the place as the trip to Makkah often is first time to majority of pilgrims, thus it becomes very difficult to reach the health centers.

The review study of [10] reported that during Hajj many pilgrims' are suffering from various infectious and chronic diseases. The major health problems encountered by pilgrims are respiratory diseases $(73.33 \%)$, heat effects $(16.67 \%)$, diabetes (13.32\%), cardiovascular (10\%), gastroenteritis (10\%), hypertension $(6.67 \%)$, and urinary tract infection including trauma $(3.33 \%)$. Whereas study of [11] observed that cardiovascular diseases is the main reason of death during Hajj.

In the last few years, new technologies have been proposed to overcome medical challenges and provide healthcare services like real-time monitoring, observing health status, managing diseases, and remote connecting to hospitals. Wireless body area network (WBAN) is one of the most promising technology that enables monitoring of health conditions, disease diagnosis, and real-time observing. The WBAN is designed as a sensor network located in patient body to collect patient medical information, and then send information to the coordinator. The coordinating or monitoring sensors search for a suitable communication network to local server to store data and then communicate with remote database server for diagnosis purpose. Until now, WBAN has been deployed for in-door based medical applications in hospitals and clinics. Hence, it is essential to ensure some of the vital requirements of quality of service of WBAN e.g. low energy consumption, higher throughput, lower delay and no collision while deploying at out-door based healthcare purposes especially for overcrowded Hajj environment. Moreover, quality of service, sensitivity of patient's information, and short time during data transmission are critical. The power consumption of WBAN keeps increased due to the growth of data rate and data transmission distance and resulting in decrement of network life.

According to [12], in crowded places like Hajj, medical data transmission must be seamless and reliable by using multi-hop based routing which provides low power consumption and consistent data routing of wireless communication. Selecting the shortest path for transferring 
data plays a key role for quality of services in terms of the power consumption and delay.

\section{RELATED WORKS}

Recently, population-based algorithms such as Ant Colony optimization (ACO), Genetic Algorithm (GA), and Particle Swarm Optimization (PSO) have been used to improve routing algorithm in WBAN; for example, PSO algorithm presented in [12] to search for optimal location of the relay node to improve radio frequency energy in WBAN. Authors in [13] used GA to optimize extracted features in WBAN in terms of latency, classification rate and packet delivery rate. It has been showed that GA optimization algorithm is effective in sensor classification. Furthermore, ACO is proposed in [14] to find shortest route through sensor node. The distance route is calculated from patient to medical center then the shortest node is selected based on Bayesian game formulation. According to [15], Bees Algorithm has been shown to be powerful optimization methods when compare it to other population-based methods [16]. The Bees Algorithm is proposed in this paper to find best path for data to reach destination within shortest time during overcrowded Hajj environment.

\section{BEES ALGORITHM OPTIMIZATION}

Bees Algorithm is a population-based search algorithm inspired from nature of honeybees to find an optimal solution. Basic Bees Algorithm is divided into four components: parameter setting, initialization, local search, and global search. Bees Algorithm in its basic form uses a set of parameters need to be set for the algorithm as shown in Table I.

Fig. 1 shows pseudo code of the Bees Algorithm. The Bees Algorithm in Step 1 start generating $\mathrm{n}$ scout randomly as initial population. Then in Step 2, the fitness is evaluated of sites explored by scout bees. The "elite bees" are selected based on the highest fitness and neighborhood search are chosen in Step 4. In Step 5, the selected sites are recruited and more bees are employed for the elite sites as well as the fitness is evaluated. In step 6, the fittest bee is selected to produce the next bee population. The remaining bees are then assigned randomly to seek for new solutions around the domain in Step 7.

TABLE I. PARAMETERS OF BEES AlgORITHM

\begin{tabular}{|l|l|}
\hline $\mathrm{Ns}$ & Scout bees \\
\hline $\mathrm{Ne}$ & Elite sites \\
\hline $\mathrm{Nb}$ & Best sites \\
\hline $\mathrm{Nre}$ & Recruited bees of ne \\
\hline $\mathrm{Nrb}$ & Recruited bees of remaining nb \\
\hline $\mathrm{Ngh}$ & Neighborhood initial size \\
\hline
\end{tabular}

1. Initialise population.

2. Evaluate sites explored by scout bees.

3. While (criterion not match)//Starting new population.

4. Select sites to search for neighborhood.

5. Recruit more bees for best sites and fitness is evaluated.

6 . Select the best fitness.

7. Distribute remaining bees randomly for searching and then evaluate fitness.

8. End.

Fig. 1. Bees algorithm pseudo code.

\section{BEES ALGORITHM OPTIMIZATION IN WBAN}

The proposed method has following steps in each iteration:

- Seek for a node randomly in search place.

- Test the condition of the node.

- Choose nodes for neighborhood search.

- Select best nodes with shortest time and evaluate fitness.

- Search randomly and keep evaluating fitness.

- Determine to continue searching or terminate the iteration.

Fig. 2 shows the proposed Bees Algorithm in WBAN.

WBANs lead to degradation of performance e.g. energy consumption, delay, throughput and collision, when the number of sensor density increase; interaction between the sensors with WBANs or interaction between the WBANs in the same or different environment increases; and the distance between WBANs and gateway increases. Hence, deploying WBANs for pilgrims health monitoring during Hajj requires extra attention. Pilgrims healthcare facility using WBAN as proposed in this paper is a dynamic procedure because the pilgrims may sometimes in motion walking, running, and sitting.

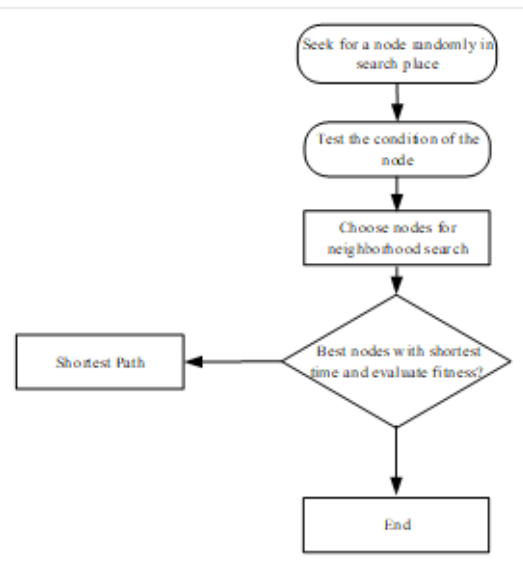

Fig. 2. Flow chart of proposed Bees Algorithm in WBAN. 


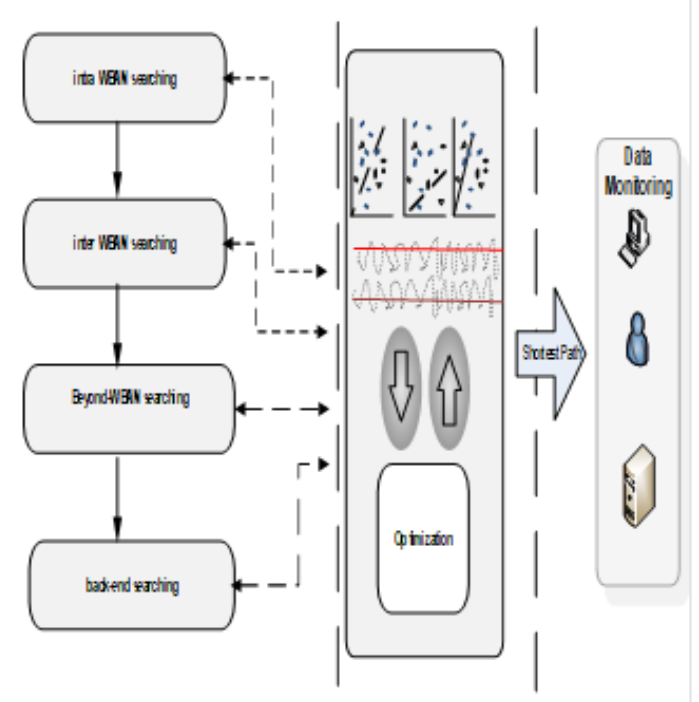

Fig. 3. Multi phases optimal search.

According to the IEEE 802.15.6 working group, nodes in WBAN are directly connected to the sink node (usually it is called as intra-WBAN and inter-WBAN) using one-hop or two-hop of star topology and thereafter the sink node which is called as coordinator is connected to access points (APs) in outer-WBAN and then to the personal server in back-end healthcare centers in a multi-hop architecture as shown in Fig. 3.

Intra-WBAN is a small network around the body can support data transmission until 1-2 meters, in some cases 2-5 meters and usually use various short-range communication infrastructure e.g. ZigBee, Bluetooth, Wi-Fi or Cellular network. And Internet is used for long-range communication to support data transmission in beyond-WBAN and back-end medical server for healthcare applications as presented in Fig. 4. Searching is performed not only in one stage but in multi phases of wireless network: intra-WBAN searching, inter-WBAN searching, beyond-WBAN searching, and backend searching.

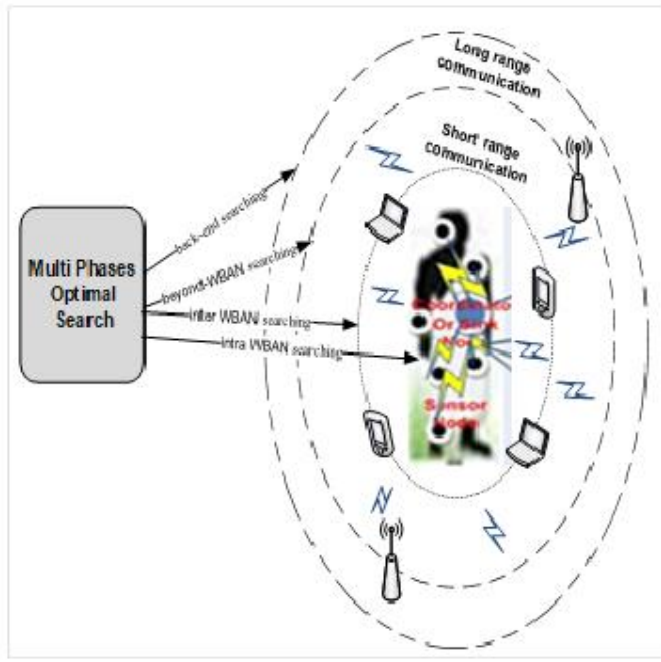

Fig. 4. Optimize long-range and short-range communications.

\section{RESULTS AND DISCUSSION}

The effectiveness of the proposed Bees Algorithm in WBAN is validated using Matlab simulator in the aspects of transmission time, energy consumption, throughput, and delay as following sub-section.

Define abbreviations and acronyms the first time they are used in the text, even after they have been defined in the abstract. Abbreviations such as IEEE, SI, MKS, CGS, sc, dc, and rms do not have to be defined. Do not use abbreviations in the title or heads unless they are unavoidable.

\section{A. Analyzing the Best Effort (the Minimum Distance/ Shortest Path or Route) from Source to Destination. the Best Cost is Measured as the Minimum Time Required for Transferring Data from Source to Destination.}

- Parameters: 1000 nodes, 30 meters distance, 20 iteration, unit of time is second.

- Description: Using these parameters, we will generate the following two graph. Fig. 5 describes the Iteration vs best cost using bees algorithm. Here we can see that the cost will be minimized if the number of iteration is increased. Fig. 6 describes the Iteration vs best cost without bees algorithm. From this graph, we can see that the cost is higher than the cost we got using bees algorithm.

The estimated data transmission time at the first five iteration with bees algorithm is presented in Fig. 7. It is shown that the transmission time decreased as the number of iteration increased. In Fig. 8, the same procedure has been followed but without applying the bees algorithms. From the Fig. 7 and 8 it is shown that the data transmission time at best cost by applying bees algorithm is less than that of without applying the bees algorithm.

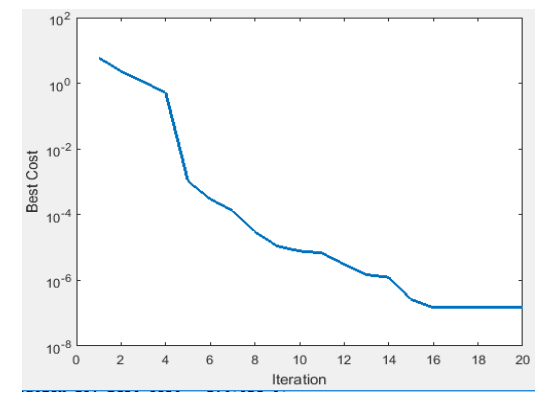

Fig. 5. Cost with applying Bees Algorithm.

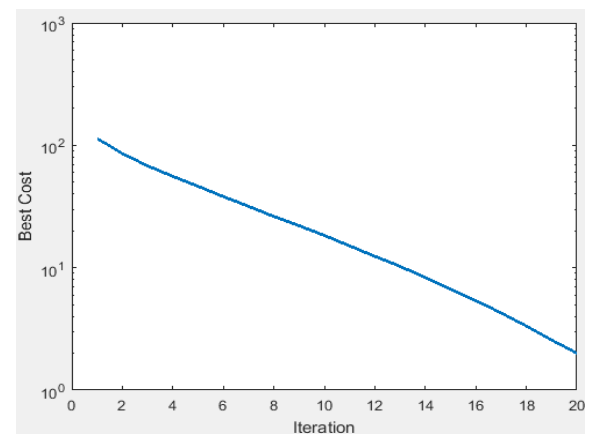

Fig. 6. Cost without applying Bees Algorithm. 
Iteration 1: Best Cost $=6.0237$

Iteration 2: Best Cost $=2.3119$

Iteration 3: Best Cost $=1.1101$

Iteration 4: Best Cost $=0.51486$

Iteration 5: Best Cost $=0.0010406$

Fig. 7. First 5 iteration with Bees Algorithm.

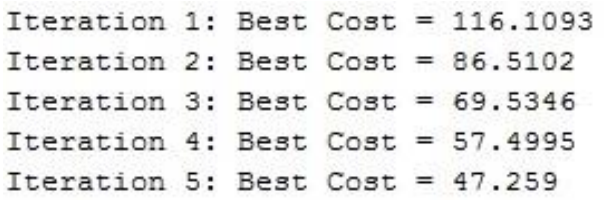

Fig. 8. First 5 iteration without Bees Algorithm.

\section{B. Analyzing the Total Amount of Energy Consumption During Packet Transmission in Minimum or Shortest Distance.}

- Parameters: 1000 nodes, 30 meters distance, 20 iteration, consider packet not bit and followed by the first analytical result.

- Description: Using these parameters, we will generate the following two graphs. Fig. 9 describes the total energy consumed during best cost by applying Bees Algorithm. Here we can see that the energy consumption will be minimized if the number of iteration is increased.

Fig. 10 describes the total energy consumed during best cost without applying Bees Algorithm. From this graph, we can see that the energy consumption is higher than the total energy consumption we got using bees algorithm.

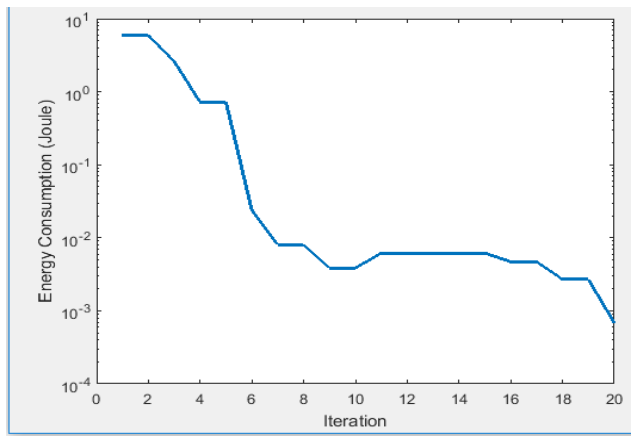

Fig. 9. Energy consumption with Applying Bees Algorithm.

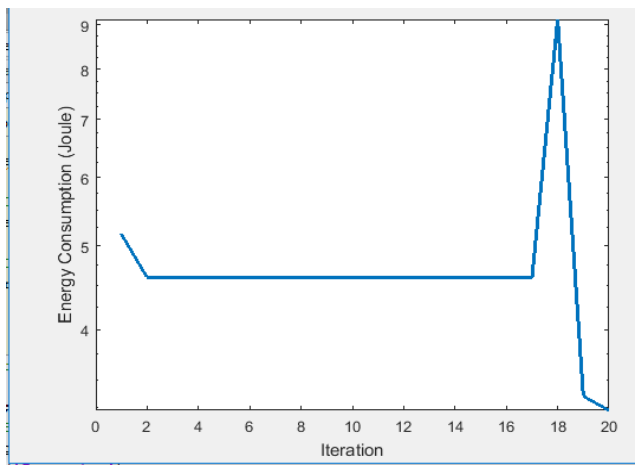

Fig. 10. Energy consumption without applying Bees Algorithm.

\section{Analyzing the Delay}

- Parameters: 1000 nodes, 30 meters distance, 20 iteration, less than $250 \mathrm{~ms}$, packet size 50-300 bytes.

- Description: Using these parameters, we will generate the following two graphs. Both the graphs show Delay vs iteration where the unit of delay is second. Fig. 11 describes the Iteration vs delay using Bees Algorithm. Here we can see that the delay will be minimized if the number of iteration is increased. Fig. 12 describes the Iteration vs delay without Bees Algorithm. From this graph, we can see that the delay is higher than the delay we got using the Bees Algorithm.

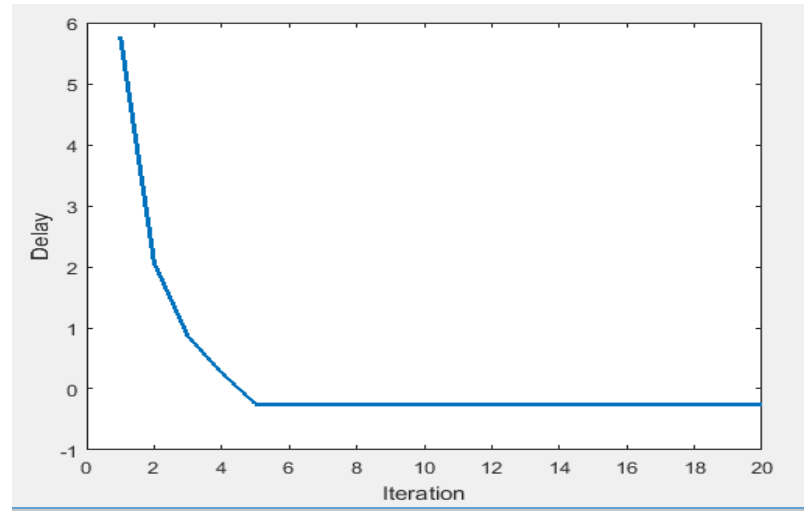

Fig. 11. Delay with applying Bees Algorithm.

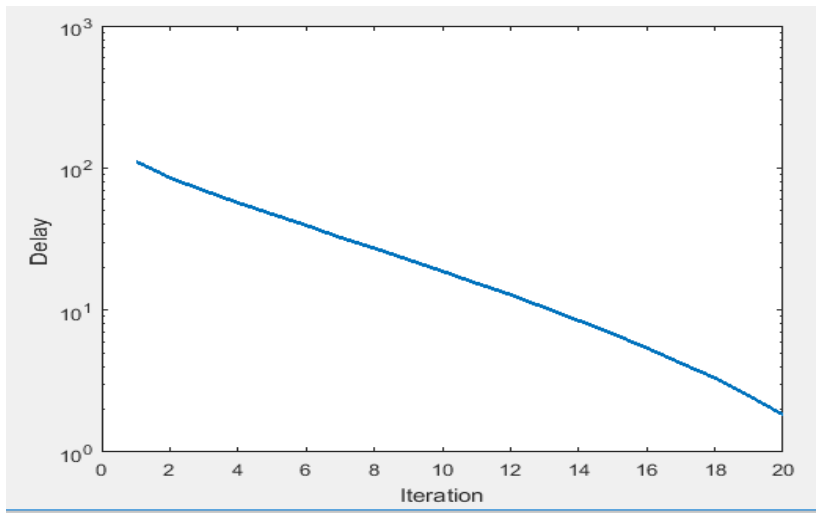

Fig. 12. Delay without applying Bees Algorithm.

\section{Analyzing Throughput}

- Parameters: 1000 nodes, 30 meters distance, 20 iteration, throughput more than $250 \mathrm{kbps}$, packet size 300 bytes.

- Description: Using these parameters, we will generate the following two graph. Both the graphs show Throughput vs iteration where the unit of throughput is kilobits per second (Kbps). Figure 13 describes the Iteration vs Throughput using bees algorithm. Here we can see that the throughput will be increased if the number of iteration is increased.

Fig. 14 describes the Iteration vs Throughput without bees algorithm. From this graph, we can see that the throughput is lower than the throughput we got using bees algorithm. 


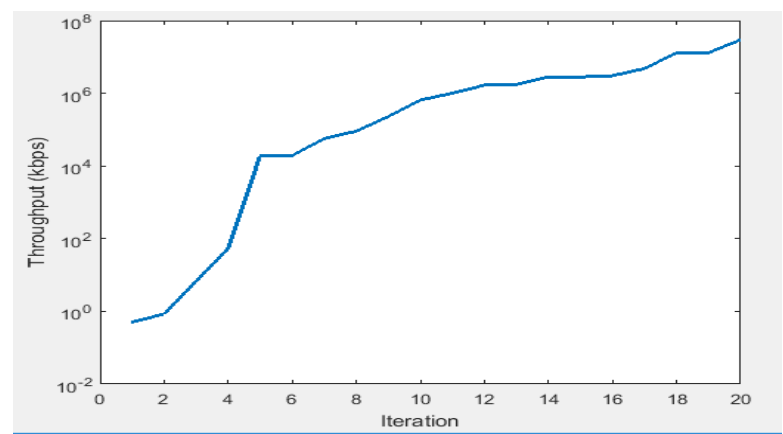

Fig. 13. Throughput with applying Bees Algorithm.

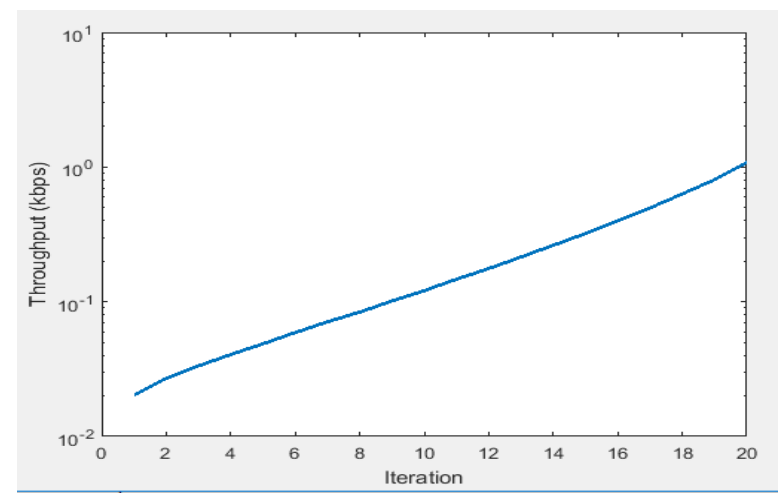

Fig. 14. Throughput without applying Bees Algorithm.

\section{CONCLUSIONS}

This paper has presented Bees Algorithm in new field of WBAN. The Bees Algorithm has briefly outlined and compared to other works. Moreover, Bees Algorithm as an optimization tool used to select shortest path in multi phases that makes data reach its destination in a shortest time with low energy consumption and less delay. Simulation results show the effective of the proposed Bees Algorithm in WBAN which is promised to be very helpful for pilgrim to overcome many challenges during Hajj.

\section{ACKNOWLEDGMENT}

This research is supported by Najran University, Najran, Kingdom of Saudi Arabia, under Research Project Code: NU/ESCI/15/028.

\section{REFERENCES}

[1] Pham, D. T., Ghanbarzadeh, A., Koç, E., Otri, S., Rahim, S., \& Zaidi, M. (2006). -The Bees Algorithm-A Novel Tool for Complex Optimisation Problems. In Intelligent Production Machines and Systems (pp. 454-459).S.R.; and Jenkins, J.E. (1982). Evaluation of component buildup methods for missile aerodynamic prediction. Journal of Spacecraft and Rocket, 19(6), 481-488.

[2] Pham, D. T., \& Castellani, M. (2009). The bees algorithm: modelling foraging behaviour to solve continuous optimization problems. Proceedings of the Institution of Mechanical Engineers, Part C: Journal of Mechanical Engineering Science, 223(12), 2919-2938.

[3] Abdullah, S., \& Alzaqebah, M. (2013). A hybrid self-adaptive bees algorithm for examination timetabling problems. Applied Soft Computing, 13(8), 3608-3620.

[4] Ali, G. A., \& Jantan, A. (2011). A new approach based on honeybee to improve intrusion detection system using neural network and bees algorithm. In International Conference on Software Engineering and Computer Systems(pp. 777-792). Springer, Berlin, Heidelberg.

[5] Pham, D. T., Koc, E., Lee, J. Y., \& Phrueksanant, J. (2007). Using the bees algorithm to schedule jobs for a machine. In Proc eighth international conference on laser metrology, CMM and machine tool performance, LAMDAMAP, Euspen, UK, Cardiff (pp. 430-439).

[6] Yuce, B., Mastrocinque, E., Lambiase, A., Packianather, M. S., \& Pham, D. T. (2014). A multi-objective supply chain optimisation using enhanced Bees Algorithm with adaptive neighbourhood search and site abandonment strategy. Swarm and Evolutionary Computation, 18, 7182.

[7] Pham, D. T., Otri, S., Afify, A., Mahmuddin, M., \& Al-Jabbouli, H. (2007). Data clustering using the bees algorithm. In 40th CIRP International Manufacturing Systems Seminar.

[8] Xu, W., Tian, S., Liu, Q., Xie, Y., Zhou, Z., \& Pham, D. T. (2016). An improved discrete bees algorithm for correlation-aware service aggregation optimization in cloud manufacturing. The International Journal of Advanced Manufacturing Technology, 84(1-4), 17-28.

[9] Haj Darwish, A., Joukhadar, A., \& Kashkash, M. (2018). Using the bees algorithm for wheeled mobile robot path planning in an indoor dynamic environment. Cogent Engineering, (just-accepted), 1426539.

[10] Al Masud, S. M. R., Bakar, A. A., \& Yussof, S. (2016). Determining the Types of Diseases and Emergency Issues in Pilgrims During Hajj: A Literature Review. statistics and information, 5(6), 7.

[11] Al Shimemeri, A. (2012). Cardiovascular disease in Hajj pilgrims. Journal of the Saudi Heart Association, 24(2), 123-127.

[12] Dhaou, I. B. (2010). Client-server network architecture for safe pilgrim journey in the Kingdom of Saudi Arabia. In Intelligent Vehicles Symposium (IV), 2010 IEEE (pp. 1043-1048). IEEE.

[13] Wu, T. Y., \& Lin, C. H. (2015). Low-SAR path discovery by particle swarm optimization algorithm in wireless body area networks. IEEE Sensors Journal, 15(2), 928-936.

[14] Kalaiselvi, K., Suresh, G. R., \& Ravi, V. (2018). Genetic algorithm based sensor node classifications in wireless body area networks (WBAN). Cluster Computing, 1-7.

[15] Latha, R., Vetrivelan, P., \& Jagannath, M. (2017). Balancing emergency message dissemination and network lifetime in wireless body area network using ant colony optimization and Bayesian game formulation. Informatics in Medicine Unlocked, 8, 60-65.

[16] Nasrinpour, H. R., Bavani, A. M., \& Teshnehlab, M. (2017). Grouped Bees Algorithm: A Grouped Version of the Bees Algorithm. Computers, 6(1), 5 . 